\title{
Observation and modelling of fast trapped ion losses due to Neoclassical Tearing Modes
}

\author{
Emanuele Poli, Manuel García-Muñoz, Hans-Ulrich \\ Fahrbach, Sibylle Günter and the ASDEX Upgrade Team \\ Max-Planck-Institut für Plasmaphysik, \\ EURATOM Association, Garching bei München, Germany
}

\begin{abstract}
Losses of trapped fast ions in the presence of low-frequency modes are observed in the ASDEX Upgrade tokamak [Fusion Science and Technology 44, 569 (2003), Special Issue on ASDEX Upgrade] during ion-cyclotron heated discharges by means of a new fast-ion-losses detector. The expulsion is explained in terms of the magnetic drift induced by the perturbation field when the ratio between the bounce frequency and the toroidal precession frequency equals the toroidal mode number.
\end{abstract}




\section{INTRODUCTION}

The loss of fast ions can affect the performance of a tokamak in several ways. First of all, the confinement of fusion products is one of the factors determining the energy balance of the discharge in reactor-grade plasmas. In addition, if the expulsion of energetic particles is sufficiently intense and localised, it can lead to damages of the plasma-facing components of the reactor. Finally, the radial transportation of fast particles can reduce the heating efficiency of schemes based on the injection of neutral beams (NBI) or ion-cyclotron (IC) waves [1]. Apart from these practical consequences, moreover, the expulsion of energetic ions is being intensively studied as it provides an insight into the magnetohydrodynamic (MHD) activity of the plasma $[2,3]$.

The theoretical investigation of the mechanisms leading to the expulsion of fast particles has focused mainly on ripple-induced losses [4, 5] and on the effect of high-frequency MHD modes [6]. In the first case, the origin of the losses is the toroidal variation of the magnetic field due to the finite number of coils. As a particle moves along the field line, its velocity parallel to the magnetic field $v_{\|}$oscillates as a result of the ripple and its radial excursion varies accordingly. These small variations average out over most of the trajectory; near the turning points, however, where these excursions are largest, they do not necessarily average to zero. This radial shift of the turning points causes the expulsion of the energetic particles. In the case of high-frequency MHD perturbations, the mode frequency equals the frequency of one of the periodic motions of the particles in the device. A typical example is given by the fishbones-induced losses, where the toroidal precession frequency of the fast trapped ions equals the mode frequency. Since the induced electric field associated to the rotation of the mode is not purely radial, but possesses also an angular component, the resonant trapped particles drift under the influence of a radial $\mathbf{E} \times \mathbf{B}$ velocity component, which is directed always in the same direction because of the phase-locking with the mode. The two mechanisms quoted above provide a good explanation for several experimental findings, as discussed also recently $[7,8]$.

As far as low-frequency $(\omega / 2 \pi \lesssim 20 \mathrm{kHz})$ MHD modes are concerned, for passing particles the formation of so-called drift islands can lead to particle losses, either because of orbit stochasticity due to the overlap of drift islands [9, 10], or because the drift islands intersect the plasma-facing components [11]. These mechanisms have been invoked as an explanation 
for the observed reduction of neutral-beam driven current in tokamaks [12] and for the losses of NBI-generated fast passing ions recently observed at ASDEX Upgrade (AUG) tokamak $[11,13,14]$.

In this paper, the focus is on the expulsion of energetic trapped particles due to lowfrequency magnetohydrodynamic perturbations. In particular, fast ions generated through IC heating (which can produce high-energy tails in the distribution function) will be considered. By means of a recently installed fast ion loss detector (FILD) in AUG, the expulsion of trapped ions with gyroradii corresponding to energies well above $E=200 \mathrm{keV}$ has been observed. FILD provides energy and pitch-angle resolved measurements of fast ion losses, with a bandwidth of $1 \mathrm{MHz}$ [13]. Its design is based on that of the $\alpha$-particle detector developed to diagnose TFTR (Tokamak Fusion Test Reactor) discharges [15, 16]. The active part of the detector is a scintillator plate contained within a cylindrical cup, which can be inserted via a movable manipulator up to a few $\mathrm{mm}$ behind the limiter slightly above the midplane. Fast ions enter the detector through an aperture in the cup and hit the scintillator. The aperture acts as a magnetic spectrometer where the strike point of the ion depends on its energy and on its pitch angle, defined as the angle between their velocity and the local magnetic field B. The scintillating surface is observed via a CCD camera, which provides a slow but highly spatially-resolved image, and by an array of 20 photomultipliers, which have a bandwidth of $1 \mathrm{MHz}$ and provide therefore a very high time resolution.

The experiments discussed here have been carried out in plasmas with Neoclassical Tearing Modes (NTM) rotating at a frequency around $10 \mathrm{kHz}$ in the laboratory frame. As the periodic motion of the energetic trapped particles occurs on a much faster time scale, no direct frequency matching between the particle motion and the rotation of the mode is possible. However, at high energies the bounce frequency

$$
\omega_{b}=\frac{v_{\perp}}{q R} \sqrt{\frac{\varepsilon}{2}}
$$

and the toroidal precession frequency

$$
\omega_{t p}=\frac{q v_{\perp}^{2}}{2 \varepsilon R^{2} \Omega_{c}}
$$

of the trapped particles become closer and closer to each other, since the former scales with the particle velocity $v$, while the latter is proportional to $v^{2}$ (in the previous formulas, $v_{\perp}$ is the velocity component perpendicular to the magnetic field, $q$ is the safety factor, $R$ is the 
major radius, $\varepsilon$ is the inverse aspect ratio and $\Omega_{c}=q_{i} B / m_{i}$ is the cyclotron frequency, $q_{i}$ and $m_{i}$ being the charge and the mass of the ion, respectively). The superposition of these two periodic motions can then give rise to a sort of "beating" that can match the mode frequency, even in the limit of vanishing mode rotation. It is interesting to note that, in this limit, no induced electric field is associated with the mode. This means that the radial displacement of trapped particle orbits in the presence of a quasi-static mode has a purely magnetic origin.

In this paper, the experimental evidence for the expulsion of fast trapped ions caused by NTMs in IC-heated plasmas is presented in Sec. II. After a brief review of the theory of mode-induced losses (Sec. III), the physical mechanism leading to the radial drift of trapped particles in the presence of quasi-static modes is explained in some detail in Sec. IV (to the best of the authors' knowledge, such an analysis is missing in the literature). Finally, the application of these results to the interpretation of the experimental data follows in Sec. V.

\section{EXPERIMENTAL RESULTS}

The experiments discussed here were performed in plasmas with toroidal plasma current $I_{p}=1.2 \mathrm{MA}$, toroidal magnetic field ramp of $B_{t}=1.95-2.15 \mathrm{~T}$ and safety factor at the edge $q_{95}=3.2$. Auxiliary plasma heating was provided by means of two NBI sources delivering a total power of $5 \mathrm{MW}$. The NBI source \#7 injects neutral deuterons with a maximum energy of $93 \mathrm{keV}$ and relative high parallel component of the velocity, while the NBI source \#3 injects neutral deuterons more radially with a maximum energy of $60 \mathrm{keV}$. In addition, 3 MW of on-axis IC heating were applied to obtain hydrogen minority heating in a deuterium plasma with a hydrogen to deuterium ratio of $n_{H} / n_{D} \approx 6 \%$. Fig. 1a shows the on-axis toroidal magnetic field ramp, $B_{t}$, the core line-integrated electron density, $n_{e}$, together with the auxiliary heating power, NBI, ICRH and ECRH (electron cyclotron resonance heating), for the reference discharge \#21081. The envelope of the normalised plasma $\beta$ during the discharge is also reported.

The MHD activity during the discharge is shown in Fig. 1b through a Fast Fourier Transform (FFT) applied to the magnetic fluctuation signal measured by a Mirnov coil [17] localized at the low field side in the vessel wall. A $(3,2)$ NTM was present in a significant time interval. EC waves were injected with the goal of stabilising the NTM (in this shot, however, 
the correct matching of the EC resonance with the island position through the magnetic-field ramp did not succeed). In order to identify the lost particles in phase space we analyse the loss pattern recorded by the CCD camera during the MHD activity. Fig. 2 shows a CCD frame for the discharge \# 21081 at $t=1.43 \mathrm{~s}$, when the NTM is present; the NTM-induced fast ion losses together with the prompt losses generated by two different NBI sources are visible. The prompt losses from the more radially injected ions (source \#3) appear in the region of higher pitch angle $\left(70^{\circ}-75^{\circ}\right)$ while a tangential source $(\# 7)$ produces prompt losses in a lower pitch angle region $\left(50^{\circ}-60^{\circ}\right)$. The pitch angle is defined as $\arccos \left(v_{\|} / v\right)$. The (3,2)-NTM contribution to the fast ion loss pattern shows a selective character at pitch angles $\left(60^{\circ}-70^{\circ}\right)$ and gyroradius corresponding to hydrogen ions with $E_{H}=600 \mathrm{keV}$ (the question about which particle species is detected by the FILD is addressed in Sec. V).

To separate the lost particles due to the NTM magnetic perturbation from the common NBI prompt losses, a FFT was applied to the signal of the photomultipliers which cover the phase space regions where NTM induced losses are detected, Fig. 3a. Tracking the main frequency of magnetic perturbation and of the fast ion losses we find, in general, a significant correlation between the NTM amplitude and the particle loss signal (Fig. 3b). An increase of the magnetic-island width when the ECRH is switched on is observed, together with a corresponding increase of the FILD signal. The decrease of this signal starting from $t \simeq 2.3 \mathrm{~s}$ can be explained in terms of change of the position of the IC resonance during the $B_{t}$ ramp, which leads to a shift of the energetic particles interacting with the NTM with respect to the mode location (see also Sec. V).

Fig. 4 shows the time traces of the fast ion loss signal and of the radial magnetic-field perturbation $\partial \tilde{B}_{r} / \partial t$ due to the rotating mode as registered by a Mirnov coil located at nearly the same toroidal position as the FIL detector. The losses are clearly modulated at the same frequency as the island rotation frequency. The maximum losses are found when $\partial \tilde{B}_{r} / \partial t=0$, which happens halfway between the $O$-point and the $X$-point of the island. This will be shown to be consistent with the expulsion mechanism discussed in this paper, see again Sec. V. 


\section{EXPULSION OF TRAPPED PARTICLES IN A TOKAMAK}

We turn now to the theoretical background of fast-ion expulsion in axisymmetric fusion devices. The magnetic field of a tokamak can be written

$$
\mathbf{B}_{0}=\nabla \psi_{t} \times \nabla \theta+\nabla \varphi \times \nabla \psi
$$

where $\psi\left(\psi_{t}\right)$ is the poloidal (toroidal) magnetic-flux coordinate, $\theta$ is the poloidal angle and $\varphi$ is the toroidal angle. Magnetic-field perturbations due to a MHD mode can be expressed as

$$
\tilde{\mathbf{B}}=\nabla \times \tilde{\alpha} \mathbf{B}_{0}
$$

where $\tilde{\alpha}$ is a scalar function of position. The function $\tilde{\alpha}$ is sufficient to exactly represent the radial magnetic-field perturbation, which is the most important component for the modification of particle orbits [18]. In the following, the spatial dependence of $\tilde{\alpha}$ is expressed as $\tilde{\alpha}=\tilde{\psi} \cos \xi$, where $\tilde{\psi}$ gives the amplitude of the perturbed magnetic flux and $\xi=m \theta-n \varphi-\omega t$ is the helical angle ( $m$ and $n$ are the poloidal and toroidal mode numbers, respectively). The change in the magnetic topology introduced by the presence of the mode can be described by introducing the helical flux

$$
\Psi_{h e}=\psi-\frac{\psi_{t}}{q_{s}}+\tilde{\psi} \cos \xi
$$

(with $q_{s}=m / n$ ), so that the total magnetic field can be expressed as

$$
\mathbf{B}=\nabla \psi_{t} \times \frac{\nabla \xi}{m}+\nabla \varphi \times \nabla \Psi_{h e}
$$

It is immediate to verify that this expression coincides with that for $\mathbf{B}_{0}$, Eq. (3), for $\tilde{\psi} \rightarrow 0$.

The equations of motion for the guiding centre of a particle in the presence of a rotating magnetic perturbation can be written employing a Hamiltonian formalism [6]. Introducing the variables $\rho_{\|} \equiv v_{\|} / \Omega_{c}$ and $\mu \equiv m_{i} v_{\perp}^{2} / 2 B$ and the electrostatic potential $\Phi$, the Hamiltonian function of the system can be written as (normalising the variables such that $\left.m_{i}=q_{i}=1\right)$

$$
H=\frac{1}{2} \rho_{\|}^{2} B^{2}+\mu B+\Phi
$$

The equations of motion can be expressed in terms of the Boozer coordinates $\psi, \theta$ and $\zeta$. In particular, because of their importance for the analysis presented in Sec. IV, the evolution 
equations for the radial coordinate and for the parallel velocity in the limit of vanishing $\Phi$ (quasi-static perturbation) and $\tilde{\psi}=$ const. are quoted here:

$$
\begin{aligned}
\dot{\psi} & =\frac{1}{D}\left[\rho_{\|} B^{2}\left(g \frac{\partial \tilde{\alpha}}{\partial \theta}-I \frac{\partial \tilde{\alpha}}{\partial \zeta}\right)\right. \\
& \left.-g\left(\rho_{\|}^{2} B+\mu\right) \frac{\partial B}{\partial \theta}\right] \\
\dot{\rho}_{\|} & =\frac{1}{D}\left[\left(I \frac{\partial \tilde{\alpha}}{\partial \zeta}-g \frac{\partial \tilde{\alpha}}{\partial \theta}\right)\left(\rho_{\|}^{2} B+\mu\right) B^{\prime}\right. \\
& \left.+\left[\left(\rho_{\|}+\tilde{\alpha}\right) g^{\prime}-1\right)\left(\rho_{\|}^{2} B+\mu\right) \frac{\partial B}{\partial \theta}\right]
\end{aligned}
$$

where the prime indicates differentiation with respect to $\psi, I$ and $g$ are the covariant poloidal and toroidal components of $\mathbf{B}$, respectively, and $D \equiv I+q g+\left(\rho_{\|}+\tilde{\alpha}\right)\left(g I^{\prime}-I g^{\prime}\right)$. The Hamiltonian equations of motion, i. e. the two equations presented above together with the evolution equations for $\theta$ and $\zeta$, can be integrated numerically, as performed for instance in the code HAGIS [19].

The detailed theory of mode-induced particle losses was developed for NBI fast ions using a simplified Hamiltonian function, which can be obained from Eq. (5) exploiting the smallness of the inverse aspect ratio [18]. As a result, it is found that secular radial motion occurs if

$$
\omega=n \omega_{t p}+N \omega_{b}
$$

for any integer $N$. The radial drift is due to a combination of $\mathbf{E} \times \mathbf{B}$ drift and the radial excursion following the magnetic lines caused by $\tilde{\mathbf{B}}$ (cf. also Sec. IV).

\section{TRAPPED PARTICLE MOTION IN A QUASI-STATIC MAGNETIC PER- TURBATION}

In the toroidally symmetric magnetic field of a tokamak, trapped particles perform a bounce motion on the low-field side. At the same time, these particles undergo a toroidal precession (in the direction of the plasma current in the case of ions) due to the magnetic ( $\nabla B$ and curvature) drift and to the magnetic shear. An example is shown in Fig. 5. For

typical thermal particles in a fusion plasma, the toroidal precession is much slower than the bounce motion, cf. Eqs. $(1,2)$. In the following, the particle orbits are integrated numerically using HAGIS. 
In the presence of a (radial) quasi-static magnetic-field perturbation, the dynamics of the trapped particles is changed essentially in two ways: a radial component in the parallel velocity appears and the parallel gradient in the mirror force is modified.

Concerning the first point, one can schematically write the radial velocity component as

$$
v_{r}=v_{r}^{\text {magn.drift }}+v_{r}^{E \times B}+v_{r}^{\tilde{B}}
$$

where the last term can be expressed as

$$
v_{r}^{\tilde{B}}=v_{\|} \frac{\tilde{B}}{B} .
$$

This term corresponds to the first term in Eq. (6), cf. also Eq. (4).

The behavior of slow (thermal) trapped ions is discussed first. For low- $n$ modes, thermal particles do not drift significantly along the island during a single bounce time, so that the perturbation field $\tilde{B}$ they experience is nearly constant during this time. Due to the different sign of the parallel velocity on the two legs of the banana orbit, the radial velocity given by Eq. (10) bounce-averages to zero for these bulk particles (modifications to magnetic drift and $\mathbf{E} \times \mathbf{B}$ drift due to the island are neglected).

For the sake of completeness, it has to be added that also when the mode rotation equals the precessional drift of fast trapped particles, as in the $n=1$ fishbone losses, the phase between the mode and the particle does not vary during a single bounce period, so that the perturbed field $\tilde{B}$ experienced by the particle is again constant during this time and the radial drift described by Eq. (10) averages to zero. At these high frequencies, however, the electric field associated to the mode is pretty high, since it is proportional to the mode frequency $\omega$. As already mentioned in Sec. I, this field is perpendicular to the perturbed flux surfaces, so it possesses an angular component proportional to $\sin \xi \nabla \xi$. This results in a radial component of the $\mathbf{E} \times \mathbf{B}$ velocity, directed inwards or outwards depending on the phase of the particle with respect to the island. This mechanism explains the fishbone-type losses of trapped particles in the presence of high-frequency modes [6].

As mentioned above, the second change of the dynamics of the trapped particles caused by the island magnetic field occurs in the mirror force, which can be written as

$$
\dot{v}_{\|}^{\text {mirror }}=\dot{v}_{\|}^{\text {mirror, } B_{0}}+\dot{v}_{\|}^{\text {mirror, } \tilde{B}}
$$

where the island-related term,

$$
\dot{v}_{\|}^{\text {mirror, } \tilde{B}}=-\frac{\mu}{B} \tilde{B} \cdot \nabla B=-\frac{\mu \tilde{B}}{B} \frac{\partial B}{\partial r},
$$


which corresponds to the first term in Eq. (7), leads to a shift of the turning points with respect to the unperturbed case.

In the case of energetic particles, the toroidal precession (and hence the drift along a low-frequency island) during one bounce time can be significant, so that the approximation $\tilde{\mathbf{B}} \approx$ const. during the bounce time made for thermal particles no longer applies. Fig. 6 is the "side view" of the motion of an energetic particle with respect to a $(3,2)$ island. The position of the island is highlighted in grey for half a toroidal turn. Dark grey corresponds to $\tilde{\mathbf{B}}>0$ ("first half" of the island), light grey to $\tilde{\mathbf{B}}<0$ ("second half"). For the parameters used in the figure, due to the large toroidal drift, the ion explores the whole island during the bounce motion. Morover, the phase between the particle and the island is chosen in such a way that the sign of the parallel velocity coincides along most of the trajectory with the sign of $\tilde{\mathbf{B}}$. This is clearly shown in Fig. 7 (where the trajectory over two bounce times is shown). In this figure, $\rho_{\|}, v_{r}^{\tilde{B}}$ and $\dot{v}_{\|}^{\text {mirror, } \tilde{B}}$ are plotted against the helical angle $\xi$ divided by $2 \pi$, to highlight again the position of the island. A complete drift along the island corresponds to a range $\Delta \xi / 2 \pi=1$. It can be seen that the radial parallel velocity defined by Eq. (10) is mainly positive during the bounce period (dotted line), resulting in a radial outward drift of the banana orbit. Moreover, the mirror acceleration due to the island (dashed curve) expressed by Eq. (12) is negative when the parallel velocity is negative and is positive in most of the part of the trajectory where the parallel velocity is positive (the term $\partial B / \partial r$ in Eq. (12) is always negative for trapped particles with $-\pi / 2 \lesssim \theta \lesssim \pi / 2$ ). This leads to an increase of the width of the banana orbit together with a shift of the bounce points. These two effects (radial drift along the perturbed field lines and increase of the parallel velocity due to the modified mirror force) contribute together to determining the exact resonance condition leading a particle to have the same phase with respect to the island after each bounce time. In fact, if this "geometric" resonance condition $n \omega_{t p}=\omega_{b}$ is satisfied, the situation described above will repeat at every bounce time, leading to a pile-up of the radial drifts and eventually to ion expulsion.

The total effect is shown in Fig. 8. It is important to note that a different choice of the inital phase between particle and island can lead to an inward drift, as is the case if the sign of $v_{\|}$and of $\tilde{\mathbf{B}}$ are opposite over most of the trajectory, cf. Eq. (10).

It should be finally stressed that the trapped-particle motion in this energy range cannot be described in terms of adiabatic invariants, since there is no clear time-scale separation 
between bounce motion and toroidal precession [20].

\section{NUMERICAL SIMULATIONS AND DISCUSSION}

In the analysis of the radial drift of fast trapped ions presented in the previous section, it has been assumed, for the sake of simplicity, that the toroidal precession frequency multiplied by the toroidal number $n$ exactly matches the bounce frequency. This implies a resonance with static $(\omega=0)$ modes. If the mode is slowly rotating, as is usually the case for NTMs, the resonance condition is restored if the mismatch between $n \omega_{t p}$ and $\omega_{b}$ equals the mode frequency. In terms of Eq. (8), the expulsion mechanism discussed here corresponds to $N=-1$ and $\omega \ll \omega_{t p} \sim \omega_{b}$.

The time needed for the expulsion of the energetic ions clearly depends on the initial conditions. Generally speaking, the bounce frequency of a hydrogen ion with an energy of the order of $600 \mathrm{keV}$ is of the order of $200 \mathrm{kHz}$ for ASDEX Upgrade parameters (major radius $R_{0}=1.65 \mathrm{~m}$, minor radius $a=0.5 \mathrm{~m}$ ). The expulsion of the trapped particle takes then $2 \pi k / \omega_{b} \approx 5 k \times 10^{-6} \mathrm{~s}$, where $k$ is the number of bounce periods before the particle is lost. Depending on the initial phase with respect to the mode, a particle can be expelled after very few or after many bounce times. In the latter case, the phase between particle and island changes during the motion, due to the fact that the resonance condition Eq. (8) is not exactly satisfied, so that the right phase for outward drift is reached after some time (in some cases, the particles are expelled after several transits through the resonance).

As it was shown in Sec. IV, the radial drift due to quasi-static modes is directed outwards if the sign of the parallel velocity matches the sign of the perturbed magnetic field associated to the island. Since the expulsion is likely to take place in the outer midplane, where the particle is at the outermost point of its "banana" trajectory (which is the case when $v_{\|}$is positive for the parameters considered here), the particle loss will occur in the region where $\tilde{\mathbf{B}}>0$, i. e. in the "dark grey" regions of Fig. 7. Remapping the helical angle $\xi$ onto the interval $[0,2 \pi]$, one can hence expect losses to take place for $\pi \lesssim \xi \lesssim 2 \pi$.

These predictions can be checked numerically by analysing the behaviour of an ensemble of trapped hydrogen ions in a realistic ASDEX-Upgrade equilibrium (the poloidal cross section is shown in Fig. 8) in the presence of a slowly rotating $\left(\omega=1.5 \times 10^{5} \mathrm{rad} / \mathrm{s}\right.$, corresponding to a frequency of approximately $24 \mathrm{kHz}$ ) mode with $m=3$ and $n=2$. 
To simulate the initial conditions of a bunch of ICRH-generated fast ions, they have been initialised taking their bounce points in a narrow layer $(\Delta R=\mp 6 \mathrm{~cm})$ located around $R_{\text {init }} \simeq 1.88 \mathrm{~cm}$. The underlying idea is that the IC heating is most efficient at the point where a particle spends most of the time during its orbits, i. e. at the turning points. The initial spread in the vertical direction is between 30 and $53 \mathrm{~cm}$ and the distribution is uniform in the toroidal direction between 0 and $\pi$ (since the mode has $n=2$ ). The energy range is $320<\mathcal{E}<700 \mathrm{keV}$. The maximum perturbation field experienced by the particles is of the order of $\tilde{B} / B \lesssim 10^{-3}$. It is stressed here that the results presented below do not aim at reproducing the details of the experimental maesurements reported in Sec. II (in particular, the initial fast ion distribution is not known; moreover, the magnetic equilibrium of the discharge(s) discussed above was not available as an input for the HAGIS code), but rather to show that the expulsion of the energetic ions according to the mechanism discussed in Sec. IV gives a correct description of the trends observed in the experiments. In these simulations, a particle is lost when it crosses the separatrix.

Fig. 9 shows the helical angle (normalised to $2 \pi$ ) at the loss position as a function of the expulsion time. Some of the particles are initialised in such a way that they are not confined, i. e. they are lost before completing a single banana orbit. These prompt losses are unformly distributed in $\xi$ and occur for $t<6 \times 10^{-6} \mathrm{~s}$. Particles which are expelled after two or more bounce times clearly lie mostly between $0.5 \lesssim \xi / 2 \pi \lesssim 1$, as expected. This kind of phase modulation of the losses is observed also in the experiments, see Sec. II (Fig. 4).

The number of lost particles as a function of the initial Larmor radius and of the angle $\alpha=\operatorname{atan}\left(v_{\perp} / v_{\|}\right)$(in degrees) is shown in Fig. 10. The variable $\alpha$ has been reconstructed from the initial position of the particle using the formula (rigorously valid only for small angles and small banana width) $v_{\|} / v_{\perp}=\operatorname{asin}\left(z_{\text {init }} / r\right) \sqrt{r / 2 R_{0}}$, with $r=\sqrt{z_{\text {init }}^{2}+\left(R_{\text {init }}-R_{0}\right)^{2}}$. It can be seen that the distribution of the losses is consistent with the experimental observations, cf. Fig. 2. An analysis of the resonance condition based on Eqs. (1-2) is not particularly instructive, since those formulas are again valid in the small-banana-width limit and are not suited for quantitative comparisons. It can be shown from the data, however, that the resonance condition discussed above is satisfied by the expelled particles, i. e., the difference $2 \omega_{t p}-\omega_{b}$ is very close to the island rotation frequency. It has to be added, however, that more energetic particles satisfying the resonance condition (8) for $N=-2$ (corresponding $\omega_{t p} \approx \omega_{b}$ ) lead to a broadening of the signal in the direction of larger gyroradii. 
Fig. 11 finally shows the scaling of the number of losses as a function of the perturbed magnetic flux. In this scan, the same ensemble of particles has been considered and only the strength of the magnetic-field perturbation has been changed. The linear dependence found in the simulations is not surprising, since we are dealing here with an expulsion mechanism of "convective" kind. A similar scan has been performed with respect to the location $R_{\text {init }}$ around which the particles are initialised, in order to simulate the shift of the resonance due to the $B_{t}$ ramp described in Sec. II. A reduction of the losses with increasing $R_{\text {init }}$, consistent with the experimental results presented in Fig. 3b, has been observed.

In the simulations presented above, hydrogen ions have been considered, as the heating scheme in the experiments discussed in Sec. II was hydrogen minority heating. The influence of deuterium on the observed signals is not so easy to ascertain for the parameters considered here. It is known that in the presence of hydrogen heating at the fundamental harmonic, a part of the IC power is absorbed by deuterium at the first harmonic. To have the same measured Larmor radius, a deuterium ion must have half the energy of the corresponding hydrogen ion. However, since at double mass and half energy both the bounce frequency and the precession frequency decrease by a factor of two, the condition $2 \omega_{t p} \approx \omega_{b}$ is preserved (the matching mode frequency changes instead by a factor 0.5). It is then possible that the signal due to both isotopes overlap. Numerical simulations confirm this hypotesis.

In summary, it has been shown in this paper that low-frequency modes like NTMs can be responsible for the expulsion of energetic trapped particles as those generated during IC heating. In this case, the matching between particle motion and mode rotation necessary to satisfy the resonance condition depends crucially on the fact that bounce motion and toroidal precession take place on comparable time scales, so that the difference of the corresponding frequencies can equal the much smaller mode frequency. The electric field connected with the island rotation has a negligible influence on this drift motion, which is mainly related to the radial drift along the perturbed magnetic-field lines. Since the direction of the drift depends on the relative phase between island position and particle position, these losses are modulated at the mode rotation frequency and occur during half of the rotation period.

As a last remark, it is interesting to observe that fusion-born $\alpha$ particles with $\mathcal{E}=3.5$ $\mathrm{MeV}$ have for ITER parameters $\left(R_{0}=6.2 \mathrm{~m}, B_{0}=5.3 \mathrm{~T}\right)$ a bounce frequency at about mid-radius $\omega_{b} / 2 \pi \simeq 460 \mathrm{kHz}$ and a precession frequency $\omega_{t p} / 2 \pi \simeq 115 \mathrm{kHz}$, indicating a potential resonance with quasi-static $n=4$ modes. 


\section{Acknowledgments}

Many useful discussions with R. Bilato, M. Maraschek, P. Martin and H. Zohm are gratefully acknowledged. 
[1] S. D. Pinches, H. L. Berk, D. N. Borba, B. N. Breizman, S. Briguglio, A. Fasoli, G. Fogaccia, M. P. Gryaznevich, V. Kiptily, M. J. Mantsinen, S. E. Sharapov, D. Testa, R. G. L. Vann, G. Vlad, F. Zonca and the JET-EFDA Contributors, Plasma Phys. Control. Fusion 46, B187 (2004).

[2] A. Fasoli, C. Gormezano, H. L. Berk, B. Breizman, S. Briguglio, D. S. Darrow, N. Gorelenkov, W. W. Heidbrink, A. Jaun, S. V. Konovalov, R. Nazikian, J.-M. Noterdaeme, S. Sharapov, K. Shinohara, D. Testa, K. Tobita, Y. Todo, G. Vlad and F. Zonca, Nucl. Fusion 47, S264 (2007).

[3] L. Chen, F. Zonca, Nucl. Fusion 47, S727 (2007).

[4] A. H. Boozer, Phys. Fluids 23, 2283 (1980).

[5] R. J. Goldston, R. B. White, A. H. Boozer, Phys. Rev. Lett. 47, 647 (1981).

[6] R. B. White, M. S. Chance, Phys. Fluids 27, 2455 (1984).

[7] M. Yoshida Y. Koide, H. Takenaga, H. Urano, N. Oyama, K. Kamiya, Y. Sakamoto, Y. Kamada and the JT-60 Team, Plasma Phys. Control. Fusion 48, 1673 (2006).

[8] S. D. Pinches, V. G. Kiptily, S. E. Sharapov, D. S. Darrow, L.-G. Eriksson, H.-U. Fahrbach, M. García-Muñoz, M. Reich, E. Strumberger, A. Werner, the ASDEX Upgrade Team and JET-EFDA Contributors, Nucl. Fusion 46, S904 (2006).

[9] S. V. Konovalov, S. V. Putvinskii, Sov. J. Plasma Phys. 14, 461 (1988).

[10] H. E. Mynick, Phys. Fluids B 5, 1471 (1993).

[11] E. Strumberger, S. Günter, E. Schwarz, C. Tichmann, New J. Phys. 10, 023017 (2008).

[12] C. B. Forest, J. R. Ferron, T. Gianakon, R. W. Harvey, W. W. Heidbrink, A. W. Hyatt, R. J. La Haye, M. Murakami, P. A. Politzer, H. E. St. John, Phys. Rev. Lett. 79, 427 (1997).

[13] M. García-Muñoz, P. Martin, H.-U. Fahrbach, M. Gobbin, S. Gnter, M. Maraschek, L. Marrelli, H. Zohm and the ASDEX Upgrade Team, Nucl. Fusion 47, L1 (2007).

[14] S. Günter, G. Conway, S. daGraça, H.-U. Fahrbach, C. Forest, M. García-Muñoz, T. Hauff, J. Hobirk, V. Igochine, F. Jenko, K. Lackner, P. Lauber, P. McCarthy, M. Maraschek, P. Martin, E. Poli, K. Sassenberg, E. Strumberger, G. Tardini, E. Wolfrum, H. Zohm and the ASDEX Upgrade Team, Nucl. Fusion 47, 920 (2007).

[15] S. J. Zweben, Nucl. Fusion 29, 825 (1989). 
[16] D. S. Darrow, H. W. Herrmann, D. W. Johnson, R. J. Marsala, R. W. Palladino, S. J. Zweben, Rev. Sci. Inst. 66, 476 (1995).

[17] M. Schittenhelm, Nucl. Fusion 37, 1255 (1997).

[18] R. B. White, R. J. Goldston, K. McGuire, A. H. Boozer, D. A. Monticello, W. Park, Phys. Fluids 26, 2958 (1983).

[19] S. D. Pinches, L. C. Appel, J. Candy, S. E. Sharapov, H. L. Berk, D. Borba, B. N. Breizman, T. C. Hender, K. I. Hopcraft, G. T. A. Huysmans and W. Kerner, Comp. Phys. Comm. 111, 133 (1998).

[20] L. G. Eriksson, F. Porcelli, Plasma Phys. Control. Fusion 43, R145 (2001). 


\section{Figure captions}

Fig. 1. (Color online) AUG discharge \#21081. Upper panel: overview of the discharge (from top to bottom, the evolution of the toroidal magnetic field, core electron density, heating power and $\beta_{N}$ are presented). Bottom panel: magnetic spectrogram showing the MHD activity.

Fig. 2. (Color online) AUG discharge \# 21081: CCD view of the light pattern produced by the incident ions ejected from the plasma due to interactions with the $(3,2)$ NTM. Prompt losses generated by both NBI sources (\#3 and \#7) are also shown.

Fig. 3. (Color online) AUG discharge \# 21081: Fourier analysis of the photomultiplier signal which is covering the area of fast ion losses at high energies.

Fig. 4. (Color online) AUG discharge \# 21081: Phase correlation between fast ion losses and the Mirnov signal $\partial \tilde{B}_{r} / \partial t$ due to the magnetic island.

Fig. 5. (Color online) "Side view" of the motion of a thermal ( $3.5 \mathrm{keV})$ hydrogen ion during $1.2 \mathrm{msec}$ (i. e. over 18 bounce periods) showing the toroidal precession of the trapped particle.

Fig. 6. (Color online) "Side view" of the motion of a fast trapped particle that satisfies the condition $n \omega_{t p}=\omega_{b}$. Due to its high energy, the particle drifts along the whole island (i. e. between $\xi=0$ and $\xi=2 \pi$ in terms of the helical angle) during a single bounce time. A mode with $n=2$ is considered.

Fig. 7. (Color online) Motion of a trapped particle that satisfies the condition $n \omega_{t p}=\omega_{b}$ as a function of the angle along the island $\xi$ (normalised to $2 \pi$ ). The solid line represents $\rho_{\|}$, whereas the dotted and dashed lines represent the terms given by Eq. (10) and Eq. (12), respectively (both multiplied by an arbitrary factor to fit on the scale). Regions of positive and negative $\tilde{B}$ are also highlighted as in Fig. 6 .

Fig. 8. (Color online) Poloidal projection (a) and time evolution of the radial coordinate

$\psi$ (b) for a particle satisfying the resonance condition $2 \omega_{t p}=\omega_{b}$. The radial drift of the particle and the shift of its bounce points can be observed. 
Fig. 9. (Color online) Helical angle $\xi / 2 \pi$ at the loss point as a function of time. The uniformly distributed losses for $t<5 \times 10^{-6} \mathrm{~s}$ are prompt losses.

Fig. 10. (Color online) Number of expelled ions as a function of the initial Larmor radius and of $\operatorname{atan}\left(v_{\perp} / v_{\|}\right)$in degrees.

Fig. 11. (Color online) Number of expelled ions as a function of the magnetic-flux perturbation showing a linear scaling with the perturbed magnetic field. 


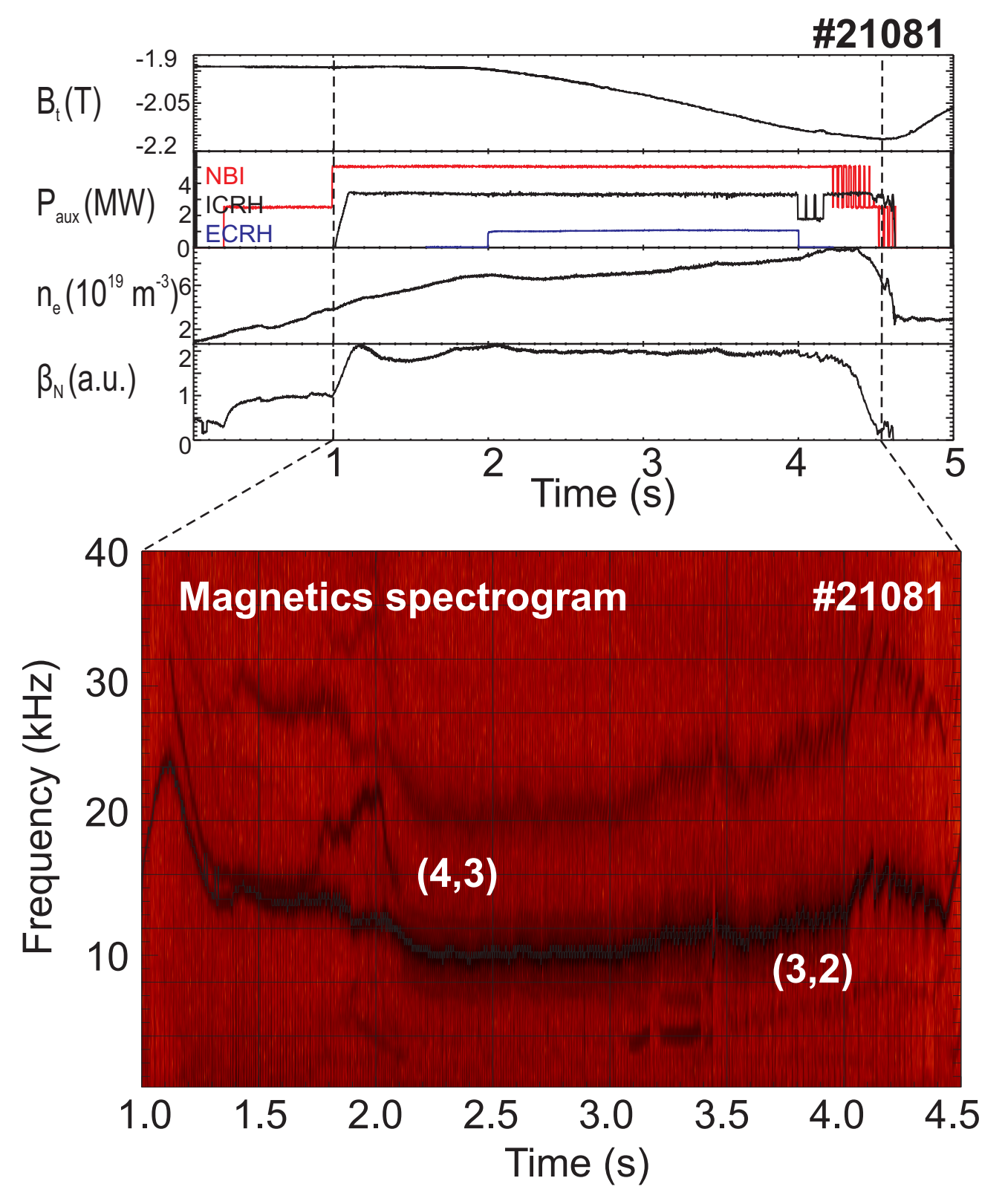

Fig. 1 


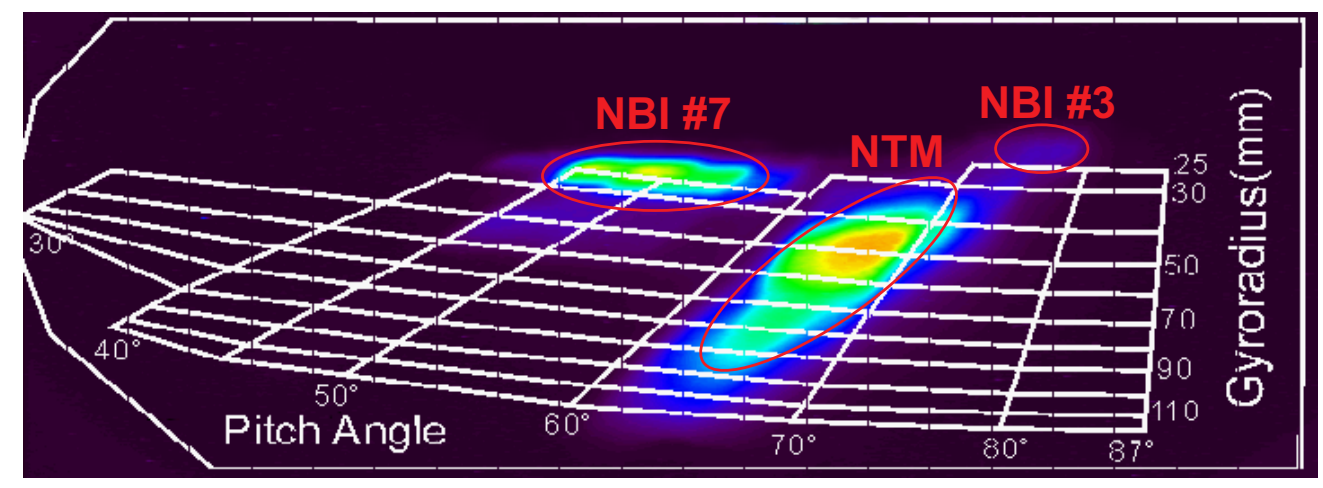

Fig. 2 


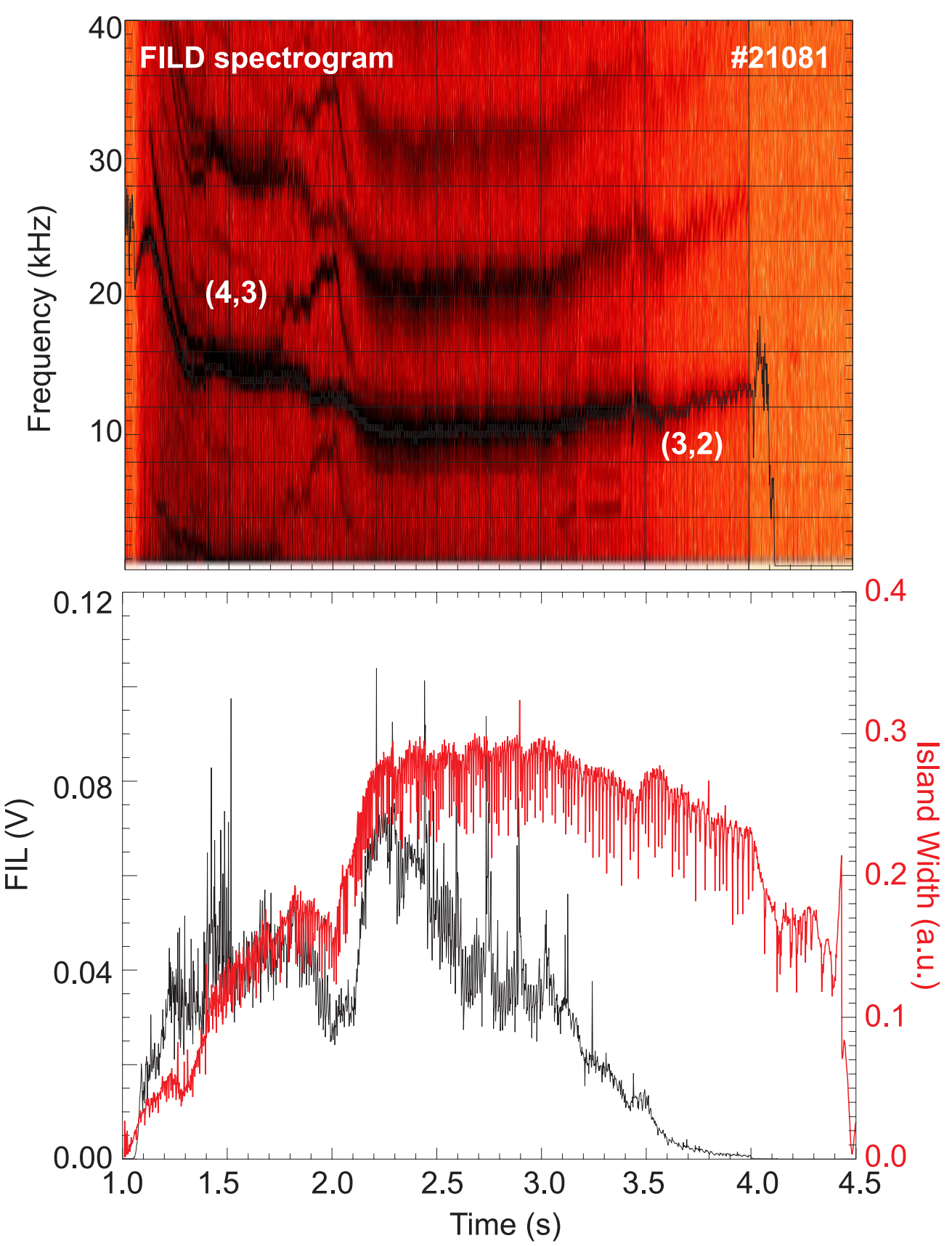

Fig. 3 


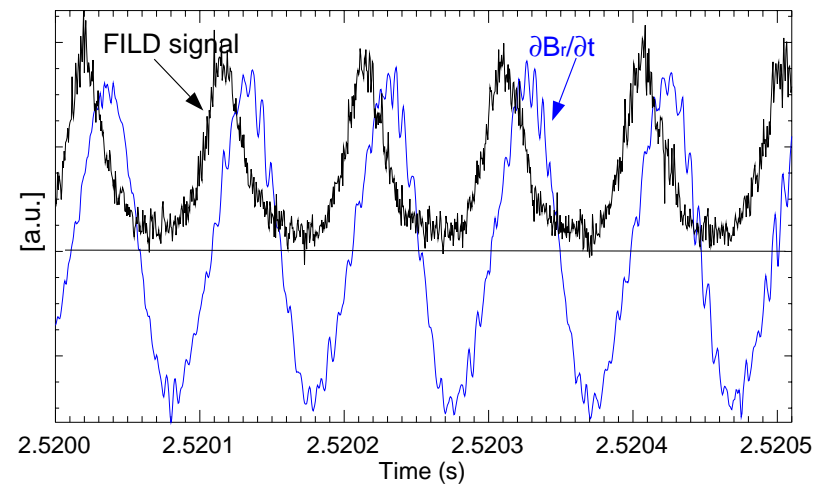

Fig. 4 


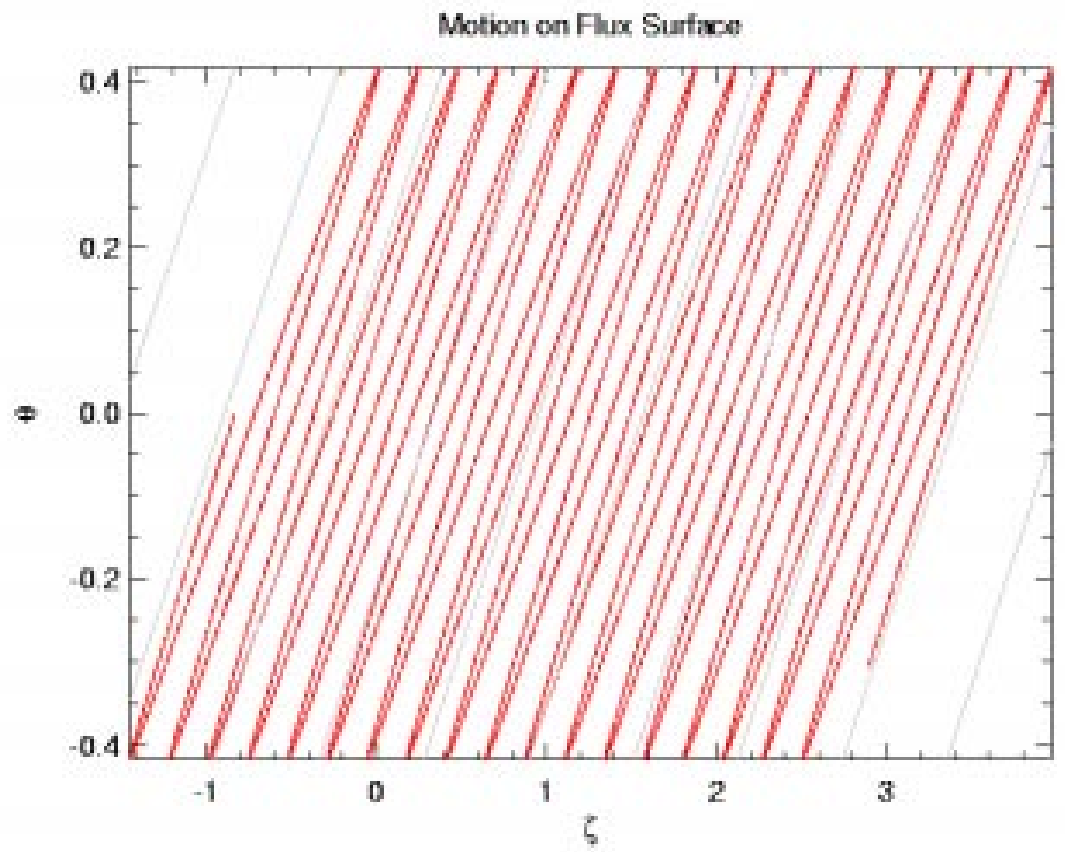

Fig. 5 


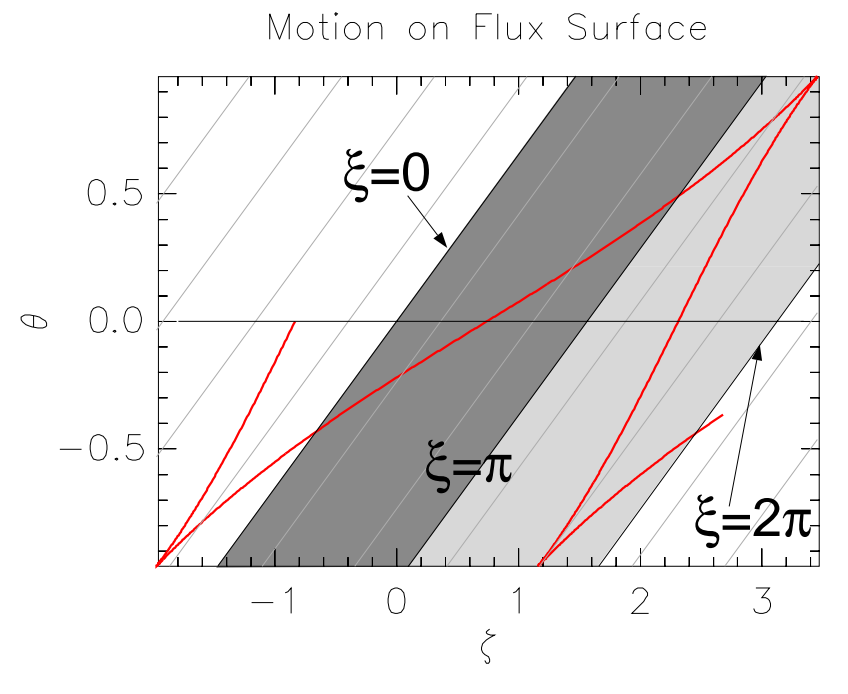

Fig. 6 


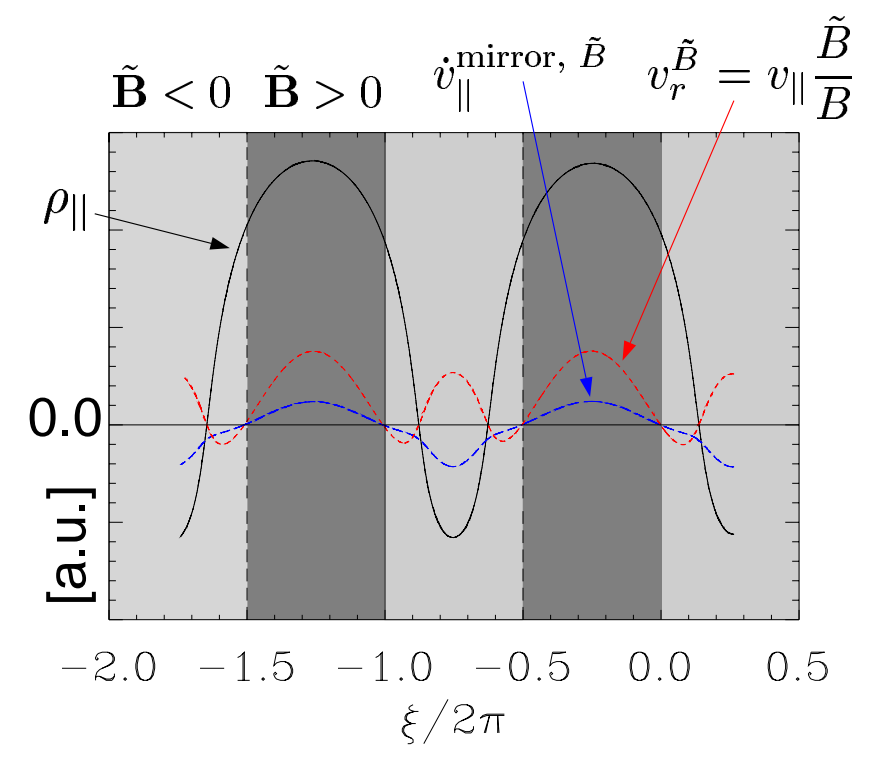

Fig. 7 
a)

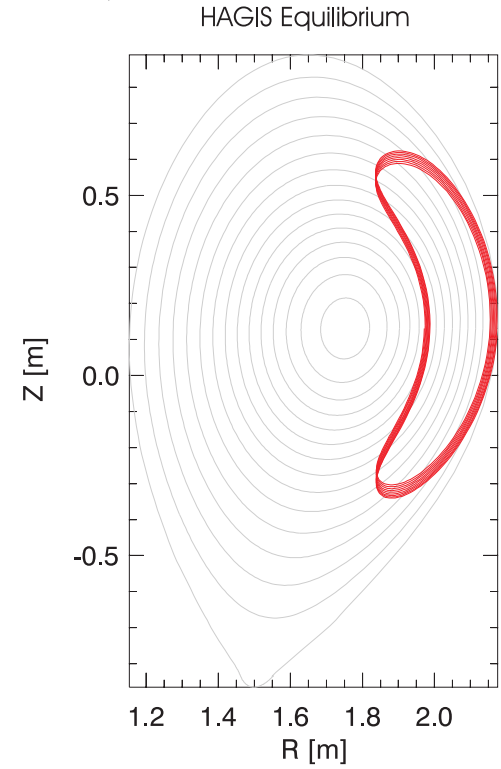

b)

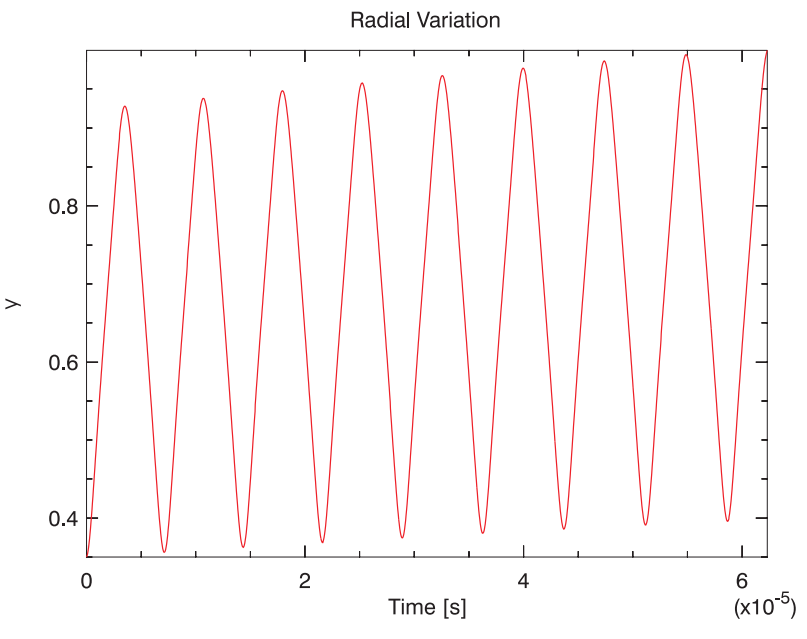

Fig. 8 


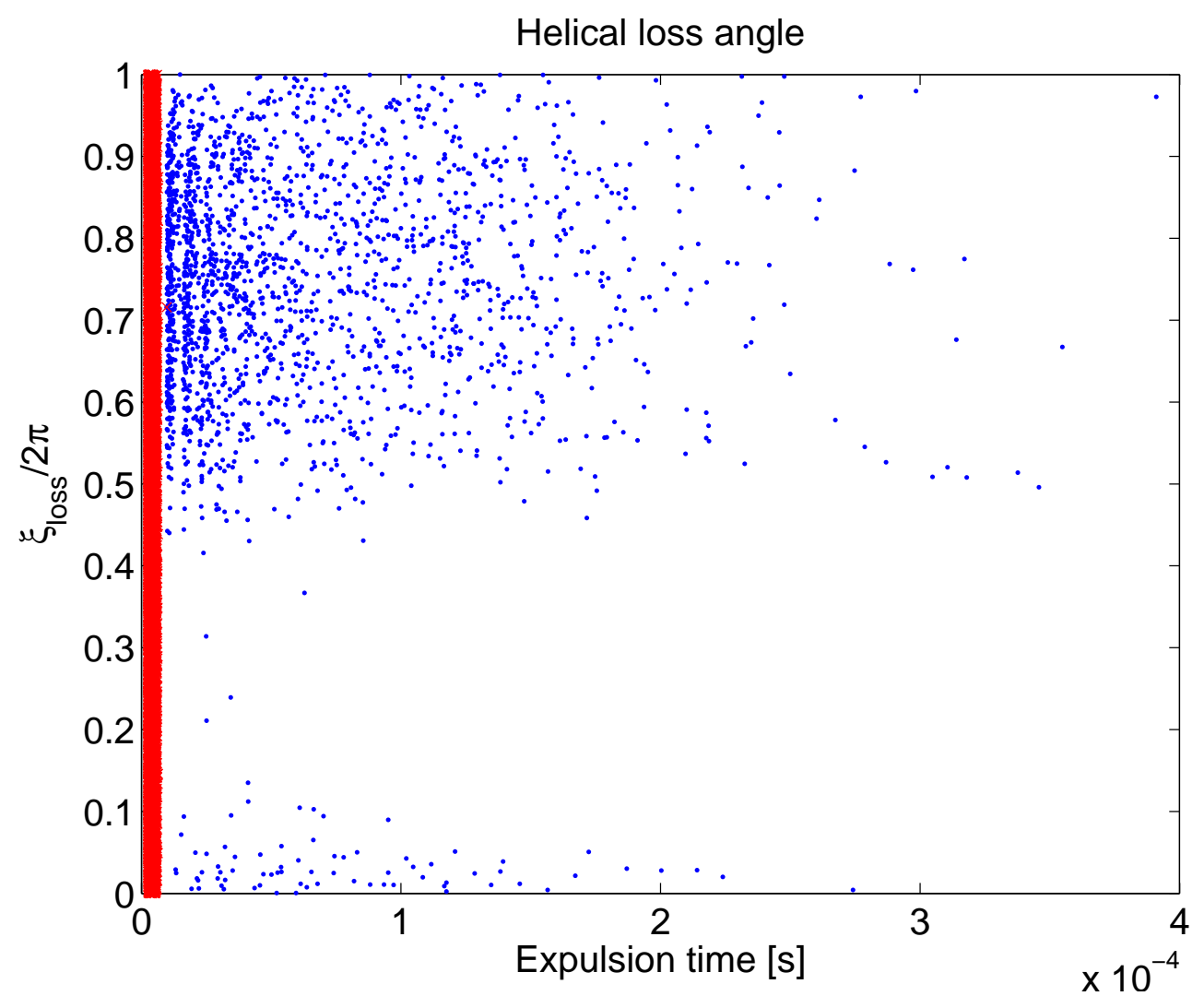

Fig. 9 
Number of counts

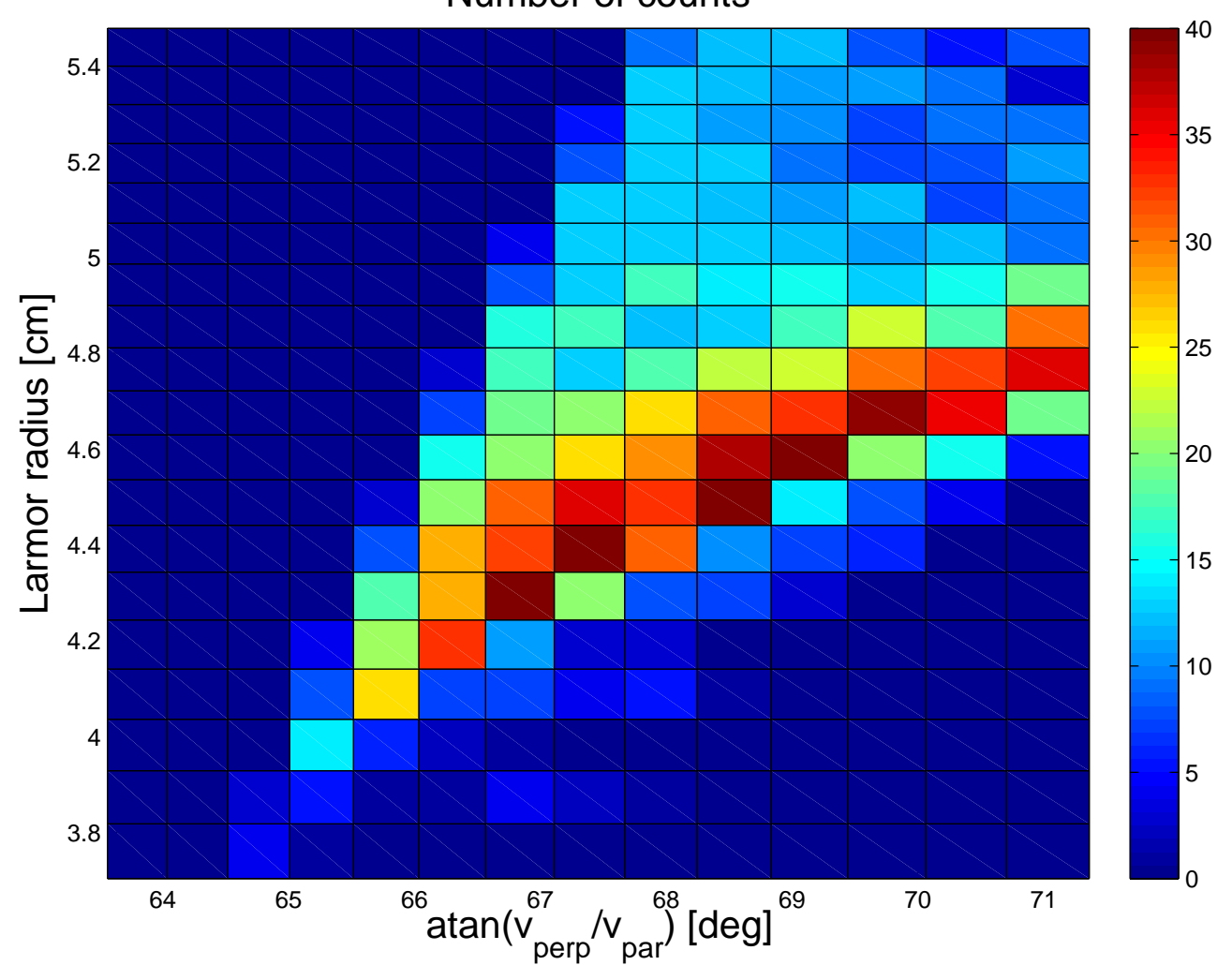

Fig. 10 


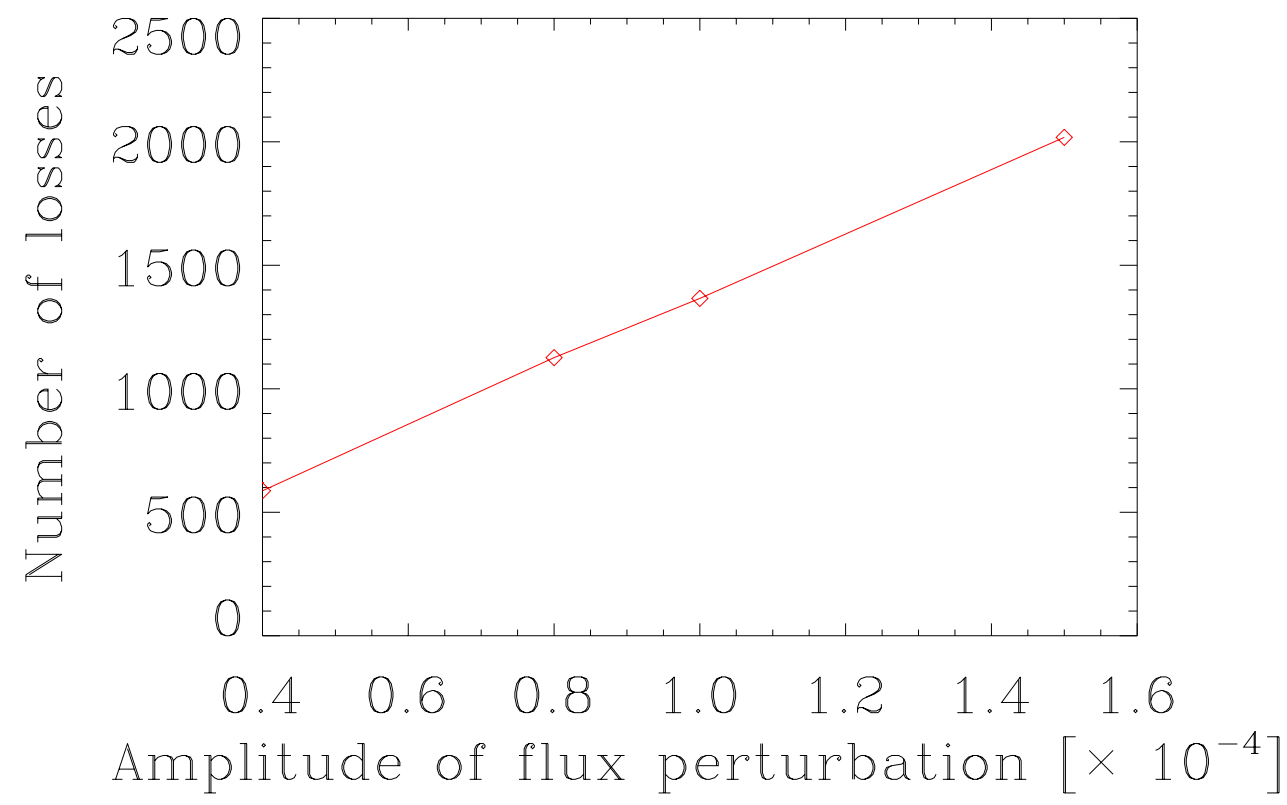

Fig. 11 